\title{
Hábitos de lectura de los estudiantes de la Universidad de Extremadura (España). Aproximación estadística
}

\author{
Ramón Pérez Parejo* \\ Álvaro Gutiérrez Cabezas* \\ José Soto Vázquez* \\ Francisco Javier Jaraíz Cabanillas* \\ José Antonio Gutiérrez Gallego**
}

Artículo recibido:

20 de junio de 2018

Artículo aceptado:

5 de diciembre de 2018

Artículo de revisión
Resumen

En el contexto de una encuesta propia sobre los hábitos lectores realizada en Extremadura entre 9212 estudiantes de todos los niveles educativos durante 2017, este estudio analiza específicamente los datos obtenidos por los estudiantes de la Universidad de Extremadura. Tras detallar el método y el campo de estudio, se han examinado distintas variables y se han desagregado los resultados en función del sexo, del área de estudios y de la distinción Grado/Posgrado.

* Departamento de Didáctica de las Ciencias Sociales, de las Lenguas y de las Literaturas, Universidad de Extremadura, España rpp@unex.es alvarogc@unex.es jsoto@unex.es jfjaraiz@gmail.com

** Departamento de Expresión Gráfica, Universidad de Extremadura, España jagutier20@gmail.com

INVESTIGACIÓN BIBLIOTECOLÓGICA, vol.33, núm. 79, abril/junio, 2019, México, ISSN: 2448-8321 pp. 119-147 
Como resultados más relevantes, más de $75 \%$ de los universitarios leen en su tiempo libre y para ello emplean algo menos de dos horas semanales a la lectura; dos tercios de ellos acuden a las bibliotecas; se sigue prefiriendo la lectura en papel; leen más las mujeres que los hombres, y los alumnos de Posgrado que los de Grado; los de Humanidades más que los de Ciencias, y dentro de la ficción los géneros literarios de misterio y aventuras son los más demandados.

Palabras clave: Lectura; Universidad; Encuesta; Bibliotecas

\section{Reading habits of Extremadura University students (Spain). Statistical approximations}

Ramón Pérez Parejo, Álvaro Gutiérrez Cabezas, José Soto Vázquez, Francisco Javier Jaraíz Caballinas and José Antonio Gutiérrez Gallego

\section{Abstract}

In the context of a survey about reading habits in Extremadura conducted bay our research group to 9212 students of all educational levels during 2017, this paper analyzes the data obtained by the students from the University of Extremadura. After giving details about the method and the field of study of the survey, variables have been examined and the results have been disaggregated according to gender, study area and the Degree/Post graduate distinction. The more relevant results are that $75 \%$ of the university students, read in their free time; they spend less than two hours a week reading; two thirds go to libraries, more to study than for loans or to consult books; reading on paper is still preferred; women read more than men; postgraduate students read more than those still studying their degree; Humanities students read more than Science students, and that within fiction, mystery and adventure genres are the most demanded.

Keywords: Reading; University; Survey; Libraries 


\section{INTRODUCCIÓN}

T as autoridades educativas y los servicios bibliotecarios universitarios reaUlizan grandes esfuerzos económicos en la adquisición de libros y en el diseño de programas de promoción de la lectura para atraer a los usuarios. Se echan en falta en ocasiones estudios estadísticos previos sobre hábitos lectores para implementar medidas concretas que ayuden a ajustar los fondos de las distintas bibliotecas universitarias a las demandas y, en consecuencia, mejorar así los índices de competencia lectora en áreas determinadas. Cualquier estudio riguroso que se aproxime a esta problemática no puede basarse sólo en análisis cuantitativos sobre el número de libros leídos, comprados o consultados, sino que debe tener en cuenta otras variables que repercuten específicamente en la lectura universitaria, tales como los soportes de lectura, las aficiones o el uso de nuevas tecnologías.

En este sentido, el objetivo principal del presente trabajo se centra en revelar las preferencias lectoras de los estudiantes de la Universidad de Extremadura $^{1}$ (UEx). Se pretende dotar de una significación estadística adecuada que se sustenta en más de 1000 encuestas realizadas durante 2017 en los distintos campus de la Universidad y en diferentes facultades correspondientes a distintas áreas de estudio a fin de establecer comparativas.

Este estudio se inscribe en el contexto de una encuesta superior sobre hábitos lectores en Extremadura realizada a algo más de 9000 estudiantes de todos los niveles educativos (Primaria, Secundaria, Bachillerato y Universidad). En este marco, el presente trabajo expone y analiza específicamente los datos obtenidos sobre los hábitos de lectura de los estudiantes de la Universidad de Extremadura con una metodología distinta con respecto a la estadística general, pues el campo de estudio, en este caso la Universidad, requiere el análisis de algunas variables específicas de los hábitos lectores de los estudiantes universitarios.

Así, tras detallar el método, los instrumentos y el campo de estudio de la encuesta, se expondrán y analizarán los datos obtenidos. Se han examinado variables como el número de ejemplares leídos, su adquisición o descarga, la dedicación semanal a la lectura, la frecuencia de visitas a las bibliotecas y su diferente uso, los géneros de lectura preferidos, los soportes utilizados, los motivos de la lectura, el uso de redes sociales o la lectura en otros idiomas,

1 Extremadura es una región del suroeste de España, limítrofe con Portugal, con 41635 kilómetros cuadrados y una población de 1079720 habitantes (datos al 1 de enero de 2017). Debido a su baja renta per cápita, una de las más bajas del país, Extremadura es la única región española que mantendrá las ayudas de los fondos de cohesión europeos. La Universidad de Extremadura (UEx) se creó en 1973. Consta de dos campus (Cáceres y Badajoz) y dos centros universitarios (Plasencia y Mérida), con un total de 19 centros. Contaba en 2014 con 24451 alumnos. 
etc. A fin de establecer comparativas significativas, se han desagregado los resultados en función del sexo, del área de estudios y de la distinción Grado/ Posgrado. Se entiende que el análisis de estas variables cubre suficientemente la especificidad de los hábitos lectores en un contexto universitario.

\section{Marco teórico y revisión de la bibliografía}

En primer lugar se expondrán los trabajos previos sobre hábitos de lectura en general antes de pasar a nombrar los que se centran en los hábitos de lectura en los niveles universitarios.

El inicio de los estudios sobre comportamientos culturales lectores se produce en la España contemporánea con las Encuestas de los bábitos de lectura que avanzó el INE (Instituto Nacional de Estadística) en 1978. Esta investigación completaba el trabajo de la Fundación Bertelsmann en varios países europeos, entre ellos España, en 1969-1994. Uno de los trabajos pioneros en la investigación sociológica de hábitos lectores regionales lo encontramos en el estudio sobre la Educación Secundaria del País Vasco (3 000 encuestas) de Olaziregi (2000: 79-93), centrado fundamentalmente en la recepción en lengua euskera y española de obras de ficción literaria. En él se confirmaban los estudios anteriores de Poulain (1988). Estudios posteriores como los de Colomer (1993) o Moret (1999) constatan el incremento lector en España hacia edades más tempranas, en torno a los 11 años de edad.

Otra investigación que completaba la panorámica regional era el desarrollado por la Fundación Germán Sánchez Ruipérez (FGSR) (2008) sobre los hábitos de lectura y compra de libros de los adolescentes extremeños entre 14 y 24 años. El informe tenía su origen en las prospecciones realizadas por la Fundación en colaboración con la Consejería de Cultura de Extremadura desde 2003. Se realizaron 600 encuestas. Un trabajo similar sería realizado en Castilla y León por esta misma institución en 2007, también en el ámbito del Plan de Fomento de la Lectura.

Igualmente interesantes resultan las conclusiones de Gil (2009) en Andalucía, con datos de 4000 familias a través de las pruebas de diagnóstico. De 2009 es el informe Hábitos de lectura y compra de libros en Castilla-La Mancha, realizado de nuevo por la Fundación Germán Sánchez Ruipérez (2010). En éste se realizaron unas 2400 entrevistas a lectores de más de 10 años, con una división territorial de poblaciones según su número de habitantes.

Dos años después, Muñoz y Hernández (2011) ayudaban a esclarecer la situación con un artículo sobre los hábitos lectores en la ESO en la provincia de Salamanca. El estudio, que constaba de 725 encuestas aleatorias entre jóvenes de 14 a 16 años, confirmaba que la lectura, aunque es valorada 
positivamente por los encuestados, no figura entre sus preferencias durante el tiempo de ocio. Mostraba un mayor índice lector en las mujeres que en los hombres.

El Ministerio de Educación, Cultura y Deporte (2015) publicó también un informe sobre hábitos lectores y prácticas culturales. De reciente aparición es el trabajo de Serna, Rodríguez y Etxaniz (2017) sobre bibliotecas escolares y su potencial como promotoras de los hábitos lectores en alumnos y familias. Con un volumen de 1528 encuestas entre niños de 8 a 12 años, concluye con la necesidad de fomentar la biblioteca escolar para mejorar los hábitos lectores, ya que su presencia per se no asegura ni incide directamente en este hábito si no viene acompañada de campañas de dinamización e integración.

En este contexto se hallan también trabajos promovidos por los gobiernos autonómicos en Cataluña (Hábitos de lectura y compra de libros en Cataluña, Departamento de Cultura de la Generalitat de Cataluña, 2016) o en la Comunidad de Madrid (Hábitos de lectura y compra de libros en la Comunidad de Madrid, Comunidad Autónoma de Madrid, 2010).

Por lo demás, la importancia de la lectura en lenguas extranjeras y el desarrollo de las secciones bilingües están fuera de toda duda (Corpas, 2014). Se hallan estudios que analizan el efecto de las enseñanzas bilingües tipo AICLE en el desarrollo de la competencia lectora (Nieto, 2018), las estrategias metacognitivas en la lectura en lenguas extranjeras (Izquierdo, 2016) o los casos en los que la lengua extranjera resulta la lengua vehicular (Muñoz y Taillefer, 2014). Todo ello ha suscitado el interés en la implementación de informes de evaluación sobre los programas de educación bilingüe por parte de instituciones como el British Council (Dobson, Pérez y Johnstone, 2010) o el Ministerio de Educación, Cultura y Deporte (2001), lo que se relaciona directamente con los objetivos de este estudio, pues la lectura en lenguas extranjeras cobra cada día más importancia en la enseñanza universitaria.

Podemos concluir que el conjunto de trabajos estadísticos actuales sobre este campo ha sido voluminoso y diverso, bien por estudios regionales sobre la Educación Secundaria (Olaziregi, 2000; Muñoz y Hernández, 2011; Molina, 2006), la lectura en red (Ministerio de Educación, Cultura y Deporte, 2013), la importancia de la participación familiar (Gil, 2009; Ministerio de Educación, Cultura y Deporte, 2014) y de los factores sociales (Moreno, Guzmán y García, 2017), sobre el sector editorial (Gremio de Libreros de Madrid, 2016; Observatorio de la lectura y el libro, 2015, 2016, 2017), que se completan con La lectura en España (Millán, 2017), entre otros.

Pasados 10 años del anterior estudio de este tipo en la región (Fundación Germán Sánchez Ruipérez, 2008), es un buen momento para retomar esta senda de trabajos de acuerdo con las peculiaridades demográficas de 
la Extremadura de 2017-2018 y su grado de ruralización, lo que afecta decisivamente a los hábitos lectores. No en vano, el Informe Pisa (Programme for International Student Asessment) (OCDE, 2016) muestra unos niveles de comprensión lectora mejorables en relación a los entornos educativos de regiones que requieren de intervención, y Extremadura es la única región española que mantendrá las ayudas de los fondos de cohesión europeos (Nieto y Cárdenas, 2015; Ramajo, Márquez y Miguel, 2014) entre cuyos objetivos se encuentra la inversión en educación e investigación (Bleotu y Doina, 2014; Furia et al., 2010; Goksu y Gungor, 2015; Grecu, Titan y Druica 2015, Grigorescu, 2012) en aras de una mayor cohesión educativa entre los territorios europeos (López y Faíña, 2014; Mate y Harris, 2014).

Como han manifestado Nieto y Gurría (2005), las poblaciones rurales van perdiendo población y servicios desde hace años y, al igual que se han estudiado variantes socioeconómicas para entender este problema (Junta de Extremadura, 2017), es necesaria la investigación de aspectos culturales y educativos que se ocupen de entidades menores, ya que las poblaciones por debajo de los 10000 habitantes suponen en Extremadura más de $96 \%$ de los municipios, con una dispersión de población que hace que, sin embargo, $50 \%$ de sus habitantes viva en localidades con más de 10000 vecinos.

Más allá de los trabajos previos sobre hábitos lectores y de las peculiaridades demográficas y económicas de la Comunidad Autónoma de Extremadura, nos anteceden diversos trabajos que conciernen específicamente a los hábitos de lectura en entornos universitarios. Se trata de estudios relativamente recientes, pues hasta ahora, como hemos dicho, las investigaciones sobre hábitos de lectura se han volcado casi exclusivamente en el análisis de segmentos de edad de lectores en formación (primaria, secundaria, bachillerato) y no en lectores adultos, entendiendo por esto mayores de 18 años, es decir, en edad universitaria, cuando la formación lectora es también clave para alcanzar la competencia lectora óptima (etapa de eficiencia lectora y perspectiva personal).

A este respecto se ha creado a nivel internacional un importante grupo de investigación llamado RIUL (Red Internacional de Universidades Lectoras, http://universidadeslectoras.org/), en el que la Universidad de Extremadura fue institución fundadora y que vuelca su investigación en la promoción de la lectura en contextos universitarios. A esta institución pertenece el libro Tendencias de la lectura en la universidad (Ramírez, 2015), que sitúa la problemática en el fomento de la lectura, las nuevas demandas y el uso de las tecnologías lectoras. La revista Álabe es el medio de difusión de la RIUL. En ella pueden leerse varios trabajos centrados en los hábitos lectores en las universidades como el de Martín (2011) o el de Martos (2010). Otros trabajos 
interesantes, que ponen el foco en cuestiones clave, son los de Echevarría y Gastón (2002), de la Escuela de Magisterio de la Universidad del País Vasco (UPV/EHU), sobre las dificultades de comprensión lectora en universitarios, así como el estudio de Arrieta et al. (2006), de la Universidad de Zulia (Venezuela), sobre la relación entre las dificultades de comprensión lectora y la redacción. En la misma línea se sitúa el artículo de Riestra (1999) sobre la reenseñanza de la escritura a alumnos universitarios.

Un estudio que se sitúa en el centro de la problemática es "Universitarios y lectura: análisis cuali-cuantitativo del uso, accesibilidad y valoración de los libros", de Gilardoni (2006), de Chile, porque examina aspectos de vital importancia para medir el alcance de los hábitos lectores en la universidad, tales como la valoración de los estudiantes sobre el acto de leer, su relación con la cultura digital, el lugar de la lectura entre sus preferencias en el tiempo libre, la frecuencia de visitas a la bibliotecas, etc.; cuestiones que nuestro estudio también ha considerado fundamentales para entender toda la problemática de la lectura universitaria. Entre sus conclusiones destacamos las siguientes: la lectura es altamente valorada por los estudiantes universitarios; en ellos se relaciona estrechamente con la función utilitaria; los libros siempre constituyen una segunda opción como alternativa de ocio; un tercio de los estudiantes admite no leer nunca; entre quienes no tienen hábito lector, se considera la lectura como un deber; los universitarios señalan que los bajos índices de lectura se deben principalmente al precio de los libros, el bajo interés por la lectura y la preferencia por otros medios de ocio, las tres razones a partes iguales. Partamos pues de este estudio que nos servirá como punto de partida y primera hipótesis de trabajo.

\section{Metodología}

\section{Fuentes}

Para el correcto desarrollo del proyecto regional de hábitos lectores ha sido necesario recurrir a un nutrido número de fuentes de información, que van desde censos oficiales y oficiosos hasta encuestas de preferencias declaradas. Los censos usados fueron muy útiles para definir la dimensión de la población objeto de estudio, así como para proceder a la adecuada estratificación del proceso de recolección de encuestas. Las encuestas se revelaron como un buen medio de acercamiento al conocimiento de los hábitos lectores de los colectivos analizados. 
En el caso del estudio concreto de los estudiantes universitarios, la información publicada por la Unidad Técnica de Evaluación y Calidad (UTEC) de la Universidad de Extremadura (UEx) ha permitido un conocimiento exhaustivo de la distribución de los alumnos en los diferentes centros universitarios. La UTEC es un servicio de la UEx que tiene como misión promover y apoyar la ejecución de la política de calidad de la misma a través de un servicio de apoyo técnico y asesoramiento al Vicerrectorado de Calidad, a los centros y a las unidades administrativas de la UEx en el diseño e implantación de sus sistemas de calidad. A través del censo de estudiantes universitarios que elabora la UTEC fue posible determinar la dimensión real de la población objeto de estudio, así como una correcta estratificación de los individuos a quienes encuestar, desagregados por campus, centro educativo, tipo de estudio y sexo (Tabla 1, p. 128).

\section{Método}

Para poder caracterizar los hábitos lectores de los estudiantes universitarios se realizó una encuesta de opinión donde se atendieron las siguientes cuestiones: centro educativo, sexo de los estudiantes, compra de libros, uso de las bibliotecas y gustos literarios.

Las fuentes principales en las que se basan las categorías objeto de la encuesta, además de los estudios propiamente estadísticos mencionados en el estado de la cuestión, han sido principalmente el estudio CONECTA de la Fundación Germán Sánchez Ruipérez (2008), en concreto sus apartados sobre situación de partida, objetivos y metodología (2008: 3-13), así como el artículo "La encuesta de hábitos de lectura. Un ensayo de estudio de comportamiento" de José Aranda (1975), director general de Estadísticas e Investigaciones Sociales del Instituto Nacional de Estadística, estudio donde se recogen los ítems que deben ser objeto de estudio en cualquier investigación estadística sobre hábitos lectores (tipo de libros, cantidad, motivaciones para el ejercicio de la lectura, gasto, materias preferidas, canales, preferencias de formato, edad y sexo, etc.), así como las razones que conducen a la selección de esos campos de estudio. El estudio además expone otros factores determinantes de la lectura relacionados con los niveles socioeconómicos, culturales y demográficos (nivel de instrucción, categoría socioeconómica, tamaño del municipio, regiones) (Aranda, 1975: 62-71).

La encuesta se realizó a los estudiantes que se encontraban en el momento de la entrevista en los centros educativos de los diferentes campus (facultades o escuelas) de ambas provincias, considerándose los siete centros del campus de Badajoz y los ocho del de Cáceres. La encuesta se desarrolló entre 
los meses de febrero y junio de 2017, los días se eligieron en función de la localización geográfica. Esta encuesta fue tanto de recolección directa en los centros mencionados como indirecta a través de la recepción de respuestas una vez que los alumnos cumplimentaban los cuestionarios en la aplicación informática Formularios de Google Drive. Para ello, se solicitó la colaboración del profesorado de la Universidad, que procedió a distribuir los cuestionarios entre sus alumnos poniendo a disposición de los mismos los Formularios en sus respectivos Espacios Virtuales.

El universo o población encuestada fue el total de estudiantes de la Universidad de Extremadura (incluyendo la Universidad de Mayores); la muestra fue tomada en los municipios extremeños donde tiene presencia la UEx.

\begin{tabular}{|c|c|c|c|c|c|c|c|c|c|c|}
\hline \multicolumn{7}{|c|}{ Total en Extremadura } & \multicolumn{7}{|c|}{ Encuestados por edades } & \\
\hline \multicolumn{1}{|c|}{ Sexo } & Número & Total & 18 & 19 & 20 & 21 & 22 & 23 & 24 & +24 \\
\hline Mujeres & 10714 & 451 & 47 & 89 & 87 & 59 & 54 & 37 & 25 & 53 \\
\hline Hombres & 8994 & 349 & 48 & 50 & 62 & 50 & 39 & 34 & 19 & 47 \\
\hline
\end{tabular}

Tabla 2. Caracterización de los encuestados por sexo y edad Fuente: elaboración propia a partir de la encuesta y UTEC

La Tabla 2 presenta el resultado de la distribución de la muestra obtenida en función de las variables edad y género, teniendo como base los datos del curso 2016-2017 de la UTEC de la Universidad. A partir del total de los estudiantes universitarios (como se presenta en la Tabla 3, p. 130) se estableció que la dimensión de la muestra total correspondía a 1300 encuestas, lo que suponía el $6.1 \%$ del total de la población de estudiantes. A continuación se muestra la fórmula empleada para el cálculo del tamaño muestral. Con el número de encuestas realizadas se garantiza que el error máximo estimado no excede de $3 \%$ para un nivel de confianza de $95 \%$ :

$$
" n "=\frac{N \cdot Z^{2} \cdot p \cdot q}{d^{2}(N-1)+Z^{2} \cdot p \cdot q}
$$

Donde:

\begin{tabular}{|ll|}
\hline Error & 0.03 \\
$\mathbf{p}$ & 0.50 \\
$\mathbf{q}$ & 0.50 \\
$\mathbf{z}$ & 1.96 \\
\hline
\end{tabular}




\begin{tabular}{|c|c|c|c|c|c|c|c|c|c|c|c|c|c|c|c|}
\hline \multirow{2}{*}{ 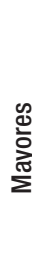 } & 造 & 0 & 0 & 0 & 0 & 0 & 0 & 0 & 0 & 0 & ๗ָ & 0 & 0 & 0 & 0 \\
\hline & 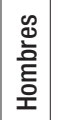 & 0 & 0 & 0 & 0 & 0 & 0 & 0 & 0 & 0 & ָ̃ & 0 & 0 & 0 & 0 \\
\hline & 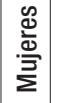 & 0 & $\stackrel{\sim}{ }$ & 尺 & $\stackrel{\circ}{\wedge}$ & $\sigma$ & 尺 & $\stackrel{\mathscr{P}}{\square}$ & $\sim$ & 0 & 0 & 0 & 0 & $\stackrel{\llcorner}{\longrightarrow}$ & $\curvearrowright$ \\
\hline & 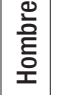 & 0 & $\stackrel{\sim}{ }$ & 字 & Ұ & $\simeq$ & 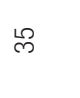 & 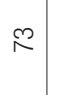 & - & 0 & 0 & 0 & 0 & $\forall$ & $m$ \\
\hline \multirow{2}{*}{ 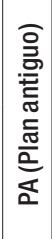 } & 总 & 0 & $m$ & 10 & 으 & 0 & 尺 & $\sim$ & m & 0 & 0 & 0 & 0 & - & 0 \\
\hline & $\mid \begin{array}{l}\mathscr{d} \\
\text { dㅎ } \\
\text { Eे } \\
\text { 호 }\end{array}$ & 0 & $\stackrel{\nabla}{\square}$ & $\approx$ & 으 & 0 & 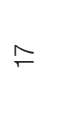 & 0 & 0 & 0 & 0 & 0 & 0 & $\sigma$ & $\sim$ \\
\hline & 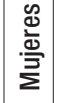 & 0 & $\underset{\sim}{\sim}$ & $\underset{\sim}{\stackrel{\Im}{\sim}}$ & $\underset{N}{\cong}$ & : & $\underset{\leftarrow}{\stackrel{\Xi}{二}}$ & $\begin{array}{l}\underset{\sim}{\sim} \\
\sim\end{array}$ & $\frac{m}{\sigma}$ & 0 & 0 & 0 & 0 & ల్లె & $\Sigma$ \\
\hline & $\begin{array}{l}\text { 흔 } \\
\text { है } \\
\text { 오 }\end{array}$ & 0 & ঙ্লি & $\stackrel{\stackrel{L}{R}}{\stackrel{N}{N}}$ & $\bar{\varnothing}$ & @్ & ळo & 苑 & 㝵 & 0 & 0 & 0 & 0 & 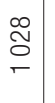 & ๗ै \\
\hline & 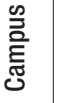 & 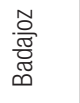 & 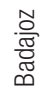 & 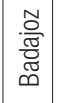 & 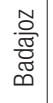 & 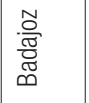 & 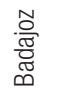 & 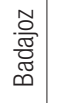 & 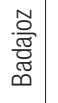 & 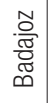 & 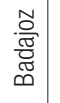 & 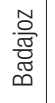 & 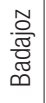 & 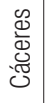 & 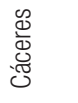 \\
\hline \multicolumn{2}{|r|}{ 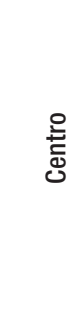 } & 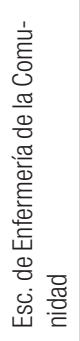 & 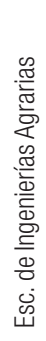 & 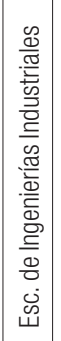 & 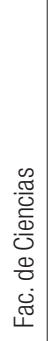 & 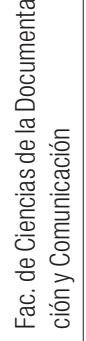 & 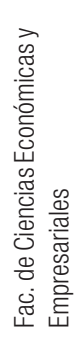 & 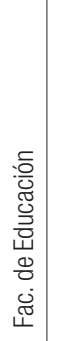 & 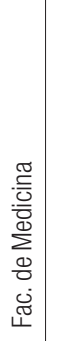 & 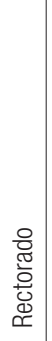 & 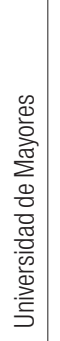 & $\stackrel{\widetilde{\Phi}}{\underline{\underline{\underline{D}}}}$ & 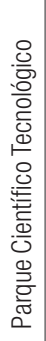 & 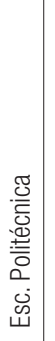 & 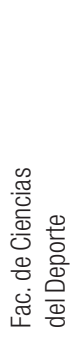 \\
\hline
\end{tabular}


HÁBITOS DE LECTURA DE LOS ESTUDIANTES DE LA UNIVERSIDAD DE EXTREMADURA...

\begin{tabular}{|c|c|c|c|c|c|c|c|c|c|c|c|c|c|c|}
\hline 0 & 0 & 0 & 0 & 0 & 0 & 0 & $\begin{array}{l}\infty \\
\stackrel{\infty}{\infty}\end{array}$ & 0 & 0 & 0 & 0 & $\infty$ & 0 & $\begin{array}{l}\infty \\
\stackrel{\infty}{L}\end{array}$ \\
\hline 0 & 0 & 0 & 0 & 0 & 0 & 0 & $\stackrel{\infty}{\stackrel{\infty}{\rho}}$ & 0 & 0 & 0 & 0 & 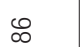 & 0 & $\begin{array}{l}\infty \\
\stackrel{\infty}{L}\end{array}$ \\
\hline$\stackrel{8}{\circledR}$ & $F$ & $\stackrel{20}{\longrightarrow}$ & $\stackrel{g}{\square}$ & 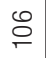 & $\nabla$ & 0 & 0 & 0 & 0 & 0 & $\sim$ & 0 & 0 & 0 \\
\hline$\stackrel{2}{\stackrel{2}{\circ}}$ & 0 & $\simeq$ & $\bar{\infty}$ & 曲 & $\nabla$ & 0 & 0 & 0 & 0 & 0 & 으 & 0 & 0 & 0 \\
\hline$\cong$ & 0 & 0 & ما & $\stackrel{\simeq}{\simeq}$ & $\infty$ & 0 & 0 & 0 & 0 & 0 & 0 & 0 & 0 & 0 \\
\hline 으 & 0 & 0 & $\sigma$ & 으 & $\curvearrowright$ & 0 & 0 & 0 & 0 & 0 & 0 & 0 & 0 & 0 \\
\hline 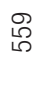 & గ్లి & $\stackrel{\stackrel{N}{N}}{\text { D }}$ & 号 & 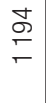 & 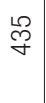 & 0 & 0 & 0 & 0 & 0 & 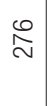 & 0 & $\begin{array}{l}\mathbb{8} \\
\mathbb{0}\end{array}$ & 0 \\
\hline$\underset{\forall}{\mathbb{F}}$ & $\underset{\sim}{\stackrel{Ð}{\sim}}$ & స్రి & న్లి & 多 & 守 & 0 & 0 & 0 & 0 & 0 & 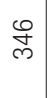 & 0 & $\stackrel{M}{\frac{M}{\gamma}}$ & 0 \\
\hline 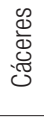 & 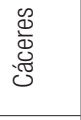 & 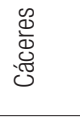 & 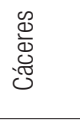 & 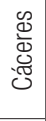 & 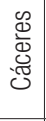 & 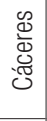 & 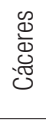 & 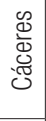 & 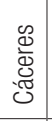 & 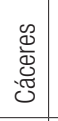 & $\frac{\pi}{\frac{\pi}{0}}$ & $\frac{\sqrt[\pi]{0}}{\sum^{\frac{\pi}{20}}}$ & $\begin{array}{l}\frac{\pi}{0} \\
\frac{0}{0} \\
\frac{\pi}{0} \\
\frac{\pi}{\alpha}\end{array}$ & $\begin{array}{l}\frac{\pi}{0} \\
\frac{0}{0} \\
\frac{\pi}{0} \\
\frac{\pi}{0}\end{array}$ \\
\hline 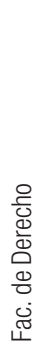 & 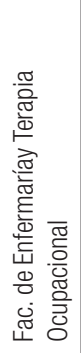 & 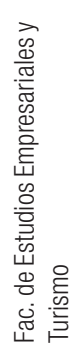 & 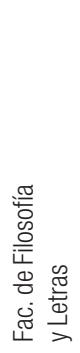 & 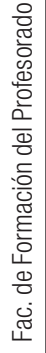 & 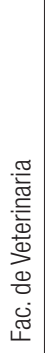 & $\begin{array}{l}\text { 음 } \\
\frac{\pi}{0} \\
\frac{0}{0} \\
\stackrel{0}{\sim}\end{array}$ & 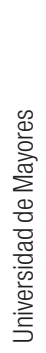 & 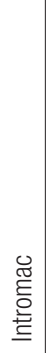 & $\underset{\mathscr{尺}}{\stackrel{\mathscr{P}}{2}}$ & 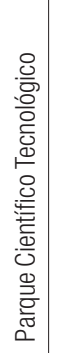 & 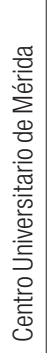 & 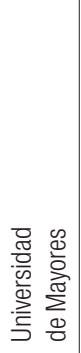 & 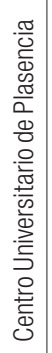 & 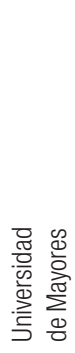 \\
\hline
\end{tabular}




\begin{tabular}{|c|c|c|c|c|c|c|c|c|c|c|c|c|c|c|c|c|c|c|c|}
\hline \multirow{4}{*}{ 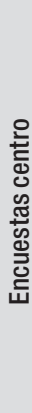 } & 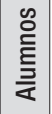 & $\stackrel{\llcorner}{\sim}$ & $\hat{m}$ & L5 & $\stackrel{\sim}{ }$ & $\bar{\infty}$ & $\Sigma$ & প্ণ & 0 & 0 & م) & $\stackrel{\infty}{-}$ & f & $\stackrel{\infty}{\sim}$ & $\stackrel{\circ}{\circ}$ & $\mathscr{子}$ & $\stackrel{\circ}{\curvearrowright}$ & $\stackrel{\sim}{\sim}$ & 0 \\
\hline & $\dot{\vec{\Sigma}}$ & $\mp$ & 0 & D & 음 & $\mathscr{\mho}$ & 요 & m & 0 & $\stackrel{\varphi}{\leftarrow}$ & $\stackrel{10}{\leftarrow}$ & $m$ & $\stackrel{\sim}{\sim}$ & $\approx$ & ৪) & $\stackrel{\sim}{\sim}$ & 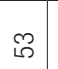 & $\stackrel{\circ}{\square}$ & 0 \\
\hline & 호 & $\Xi$ & চ & $\stackrel{2}{\sim}$ & 으 & $\stackrel{\infty}{m}$ & $\bar{N}$ & $\stackrel{\varphi}{ }$ & 0 & $\stackrel{\varphi}{\leftarrow}$ & $\underset{\forall}{\forall}$ & $\stackrel{2}{\longrightarrow}$ & $\bar{\sim}$ & 0 & $\stackrel{\sim}{\sim}$ & 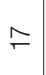 & $\widetilde{N}$ & & 0 \\
\hline & 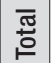 & $\stackrel{\llcorner}{\sim}$ & $\hat{m}$ & $\dot{5}$ & $\stackrel{\curvearrowright}{ }$ & $\Phi$ & 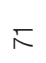 & g & 0 & $\widetilde{ల}$ & 。 & $\stackrel{\infty}{-}$ & f & $\stackrel{\infty}{\sim}$ & $\stackrel{\circ}{\circ}$ & $\stackrel{\mathscr{\checkmark}}{\forall}$ & 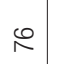 & $\stackrel{N}{ }$ & 0 \\
\hline \multirow[t]{2}{*}{ ळ } & $\dot{\vec{\Sigma}}$ & 0 & 0 & 0 & 0 & 0 & 0 & 0 & 0 & $\stackrel{\varphi}{ }$ & 0 & 0 & 0 & 0 & 0 & 0 & 0 & 0 & 0 \\
\hline & $\begin{array}{l}\text { ்ㅗ } \\
\text { 오 }\end{array}$ & 0 & 0 & 0 & 0 & 0 & 0 & 0 & 0 & $\stackrel{\varphi}{\sim}$ & 0 & 0 & 0 & 0 & 0 & 0 & 0 & 0 & 0 \\
\hline \multirow{2}{*}{ 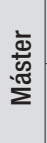 } & $\stackrel{\overrightarrow{\vec{\Sigma}}}{\Sigma}$ & - & - & $m$ & 0 & - & م & 0 & 0 & 0 & - & 0 & $m$ & 0 & - & 0 & $\nabla$ & 0 & 0 \\
\hline & 호 & - & $\sim$ & $\sim$ & 0 & - & $m$ & 0 & 0 & 0 & $\sim$ & - & $m$ & 0 & - & $\nabla$ & m & 0 & 0 \\
\hline \multirow{2}{*}{ 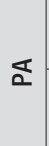 } & $\dot{\vec{\Sigma}}$ & 0 & 0 & 0 & 0 & - & 0 & 0 & 0 & 0 & 0 & 0 & 0 & 0 & 0 & 0 & 0 & 0 & 0 \\
\hline & 호 & - & - & 0 & 0 & - & 0 & 0 & 0 & 0 & 0 & 0 & - & 0 & 0 & 0 & 0 & 0 & 0 \\
\hline \multirow{3}{*}{$\begin{array}{l}\text { 을 } \\
\frac{\pi}{0}\end{array}$} & $\dot{\overrightarrow{\vec{\Sigma}}}$ & 으 & L & $\stackrel{\sim}{\sim}$ & 으 & テ & $\stackrel{8}{q}$ & m్ల & 0 & 0 & 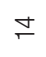 & $m$ & 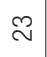 & $\approx$ & $\stackrel{\sim}{\sim}$ & $\stackrel{\sim}{\sim}$ & 守 & $\stackrel{ }{\square}$ & 0 \\
\hline & 호 & $\simeq$ & $\stackrel{\infty}{\sim}$ & 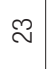 & 으 & $\stackrel{m}{ }$ & $\stackrel{\infty}{-}$ & $\stackrel{\varphi}{\square}$ & 0 & 0 & $\underset{\forall}{\Im}$ & 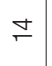 & 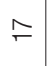 & 0 & $\stackrel{\llcorner}{\sim}$ & $\stackrel{m}{\square}$ & 尺 & 음 & 0 \\
\hline & & ৫ & ๔ & ๘ & 凹 & ఏ & 凹 & ๙ & § & ৫్ & U & ن & ভ & U & U & U & U & U & U \\
\hline & 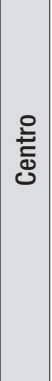 & 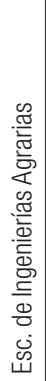 & 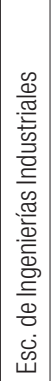 & 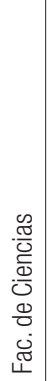 & 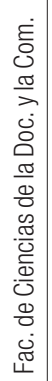 & 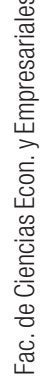 & 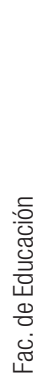 & 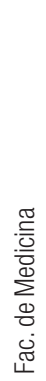 & 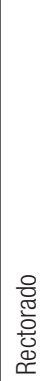 & 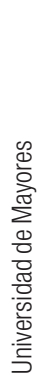 & 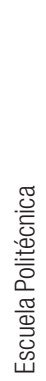 & 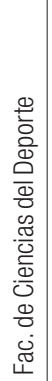 & 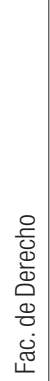 & 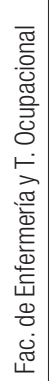 & 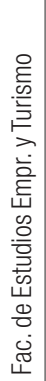 & 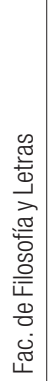 & 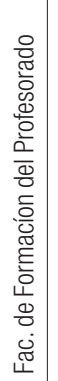 & 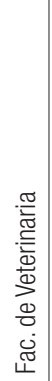 & 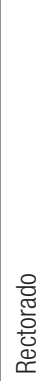 \\
\hline
\end{tabular}


HÁBITOS DE LECTURA DE LOS ESTUDIANTES DE LA UNIVERSIDAD DE EXTREMADURA...

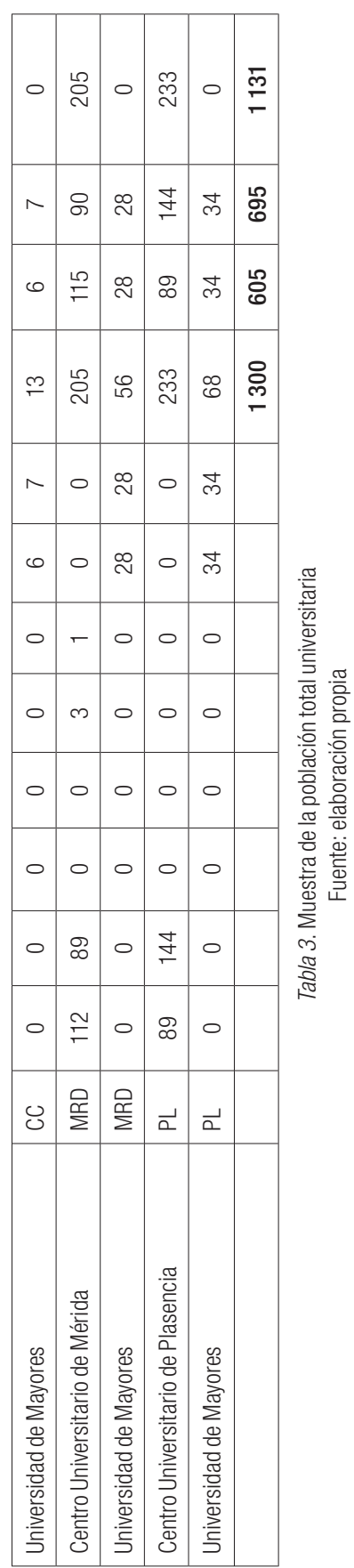


El cuestionario propiamente dicho fue configurado a partir de las necesidades de información definidas por el Proyecto de Hábitos Lectores de la Comunidad de Extremadura, siendo testado en febrero de 2017 a través de encuestados elegidos aleatoriamente en los diferentes campus con el objetivo de probar la adecuación del cuestionario, la identificación de eventuales dificultades en la comprensión de las cuestiones y verificar el tiempo medio previsto para la realización de la entrevista. En cuanto a la descripción del método de recolección, podemos indicar que todos los individuos de 17-18 y más años que cursan sus estudios universitarios en la Universidad de Extremadura eran representativos, relativamente a los fundamentos propuestos para esta operación estadística. Es necesario destacar que al mostrar los datos no se ha tenido en cuenta ningún resultado que obtuviera un valor inferior a 25 respuestas. La encuesta se desarrolló entre febrero y junio de 2017.

Después de realizar todas las encuestas mediante la aplicación de Formularios de Google Drive, la hoja de cálculos generada automáticamente por la misma era importada desde Access (utilizado como gestor de base de datos). La información era analizada a través de medidas de estadística descriptiva, las variables finales consideradas fueron las siguientes: 1) las relacionadas con el perfil biográfico del encuestado: sexo, edad, nacionalidad, nivel de estudios, centro de estudios, provincia de residencia y municipio de residencia; y 2) las vinculadas a los hábitos lectores: lectura de libros mensual, causa de la lectura, tiempo que se dedica a la lectura, consulta y uso de bibliotecas tanto universitarias como no universitarias, géneros de lectura favoritos, formato de lectura, la lectura en el tiempo libre, idioma de lectura, uso de redes sociales, compra de libros y descarga de libros.

\section{PRESENTACión y ANÁLISIS DE LOS RESUltados}

\section{Sexo}

A continuación se expondrán los datos obtenidos. En primer lugar se muestran los datos generales diferenciándolos por sexo.

El porcentaje de hombres que en este nivel no lee ningún libro al mes es mayor que el de las mujeres en más de 15 puntos porcentuales, cifra suficientemente significativa. Además, el rango entre dos y cuatro libros leídos al mes, que puede considerarse óptimo, favorece también claramente a las mujeres, con 12 puntos sobre los varones. 

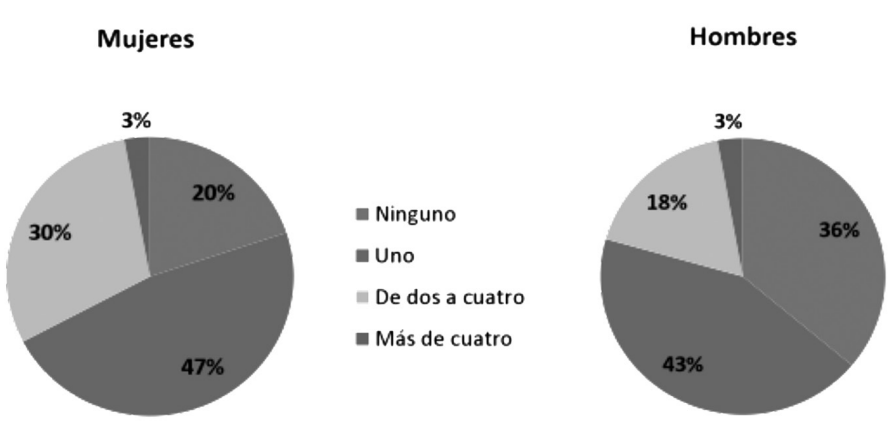

Figura 1. Libros leídos al mes por sexo Fuente: elaboración propia a partir de la encuesta

Lo mismo ocurre en la Figura 2, con unas cifras que suelen beneficiar a las mujeres, tanto en los mínimos como en los máximos. Así, es superior (cuatro puntos de diferencia) el porcentaje de hombres que no lee nada con respecto a las mujeres; y por otro lado, entre los más aficionados a la lectura también dominan las mujeres, como podemos apreciar en los apartados de "de cuatro a seis horas" y de "más de seis horas".

\section{Mujeres}

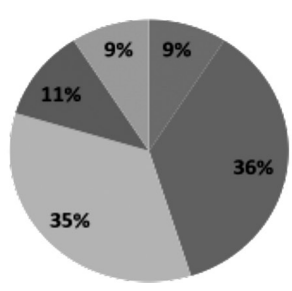

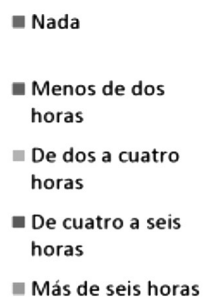

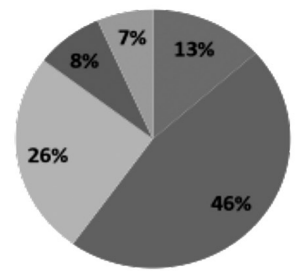

Figura 2. Tiempo dedicado a la lectura a la semana por sexo Fuente: elaboración propia a partir de la encuesta

Se ha considerado conveniente analizar la frecuencia de visitas a las bibliotecas, pues está directamente relacionada con los hábitos lectores, especialmente en niveles como estos en los que los libros suelen ser muy caros y se impone la consulta continuada de material bibliográfico y de consulta. Observamos que aproximadamente un tercio de los estudiantes universitarios no acude nunca a las bibliotecas universitarias, lo cual coincide con los resultados obtenidos por Gilardoni (2006) para los estudiantes de Chile, 
como hemos comentado anteriormente. Si cruzamos variables en relación al distinto comportamiento entre los sexos en este apartado, se observa en general la misma tónica: aunque el porcentaje de alumnos que acude más de cuatro veces a la biblioteca universitaria sea igual en ambos sexos, el porcentaje de los que no van nunca es $4 \%$ mayor en los hombres que en las mujeres (Figura 3).

Mujeres
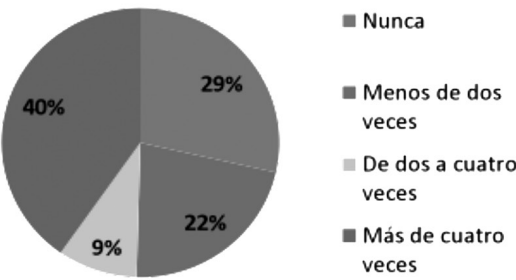

Figura 3. Frecuencia con que se acude a la biblioteca universitaria al mes (división por sexo) Fuente: elaboración propia a partir de la encuesta
Hombres

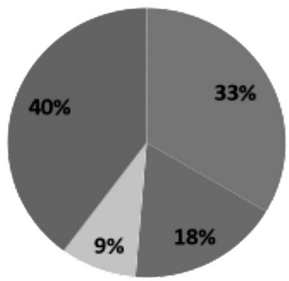

Lo que más llama la atención es que el mayor uso que se da a las bibliotecas en general es como lugar de estudio (Figura 4). Para consulta de libros acude sólo un cuarto de los encuestados. El cruce de variables en cuanto a las diferencias de comportamiento entre sexos en este apartado refleja que hay $8 \%$ más de mujeres que de hombres que usan la biblioteca para intercambio de libros, es el dato más significativo. En el resto de ítems no se observan diferencias dignas de comentario.

Mujeres

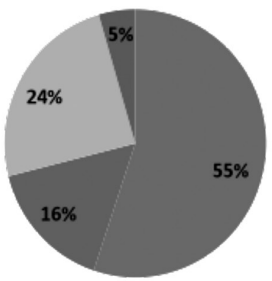

Estudio

- Intercambio de libros

- Consulta de

libros

= Internet
Hombres

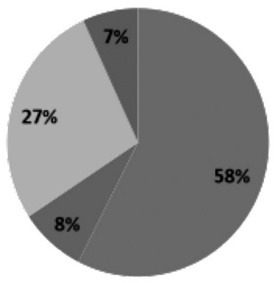

Figura 4. Uso que se le da a la biblioteca por sexo

Fuente: elaboración propia a partir de la encuesta 
La Figura 5 muestra que en los niveles universitarios dominan las lecturas disciplinares con respecto a la ficción literaria, que sólo supone 14 \% del total. Las distintas áreas disciplinares se reparten el conjunto de las lecturas. Si se aplican cruces de variables en cuanto al género en este apartado, sólo en los libros de ciencias sociales y jurídicas hallamos idénticos resultados. En los demás se observan sensibles diferencias. Así, los hombres tienen más predilección que las mujeres por libros de ciencias biomédicas, humanidades y ficción literaria, mientras que las mujeres sienten más predilección que los hombres por los libros de tecnología. Los resultados en este sentido pueden no ajustarse a lo esperado, pero sí a las encuestas realizadas. Lo decimos porque el cruce de variables refleja una mayor presencia de alumnado femenino en los grados de Humanidades, en principio más proclives a la lectura de géneros de ficción.
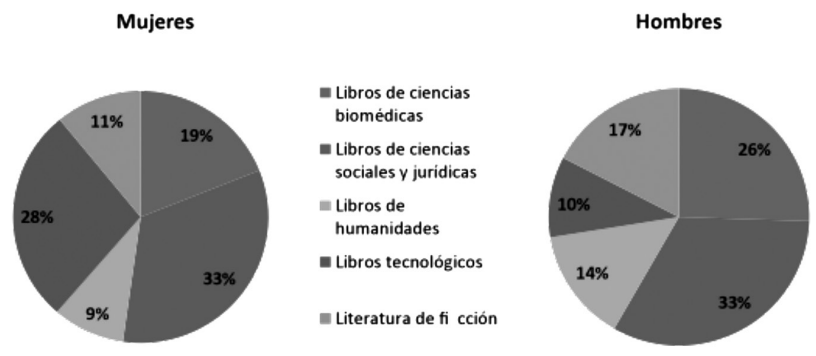

Figura 5. Temática favorita por sexo

Fuente: elaboración propia a partir de la encuesta

Según observamos en la Figura 6, dentro de los géneros ficcionales la gran diferencia se ubica en la novela romántica, siendo seleccionada mayoritariamente por mujeres, con una diferencia de $45 \%$. Las mujeres también han seleccionado más los libros de misterio, aunque este género también obtiene un alto porcentaje en los hombres. Los varones han seleccionado más los libros de ciencia-ficción, con una diferencia de $18 \%$ con respecto a las mujeres.

A las mujeres no les seducen los géneros bélico y de terror, mientras que la mayoría de los hombres afirman que a ellos no les gustan los libros románticos. El género lírico no es muy valorado por ambos sexos. 
Mujeres

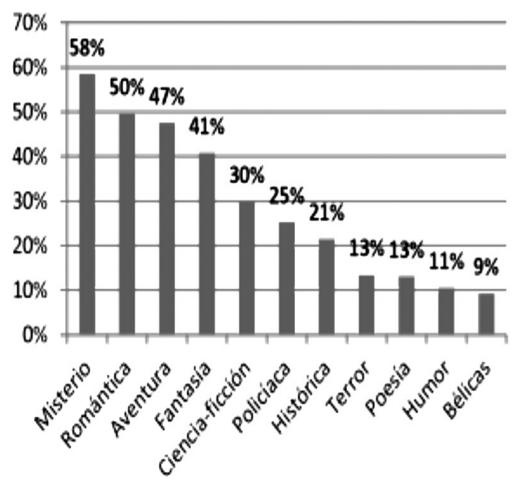

Hombres

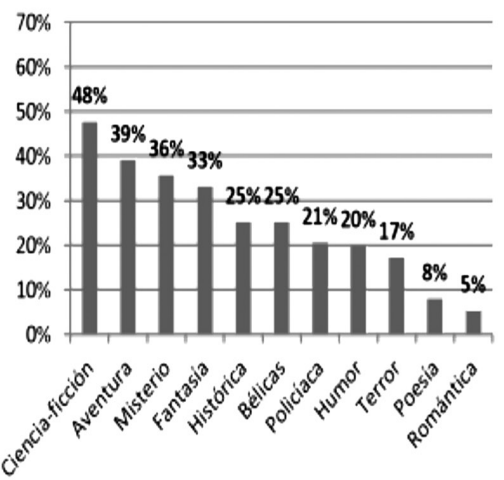

Figura 6. Temática de ficción favorita por sexo Fuente: elaboración propia a partir de la encuesta

El porcentaje de encuestados que no compra ningún libro al año es $10 \%$ mayor en los hombres (Figura 7). Es probablemente el dato más llamativo. Al margen de ello, también hay $3 \%$ de mujeres más que de hombres que compran más de 10 libros al año. Por otro lado, 42 \% de mujeres afirma comprar más de cinco libros al año; este segmento supone sólo 30 \% en los hombres. Las diferencias, pues, resultan bastante significativas tanto si analizamos los datos aisladamente como si los analizamos por grupos de respuestas. Si aplicamos el cruce de variables, estos datos concuerdan con los primeros que se expusieron acerca del número de libros leídos por sexo.

Aunque, como hemos visto, las mujeres leen más en formato papel y los hombres presentan una tendencia mayor a usar tecnologías en la lectura, lo cierto es que el número de descargas sigue siendo favorable a las mujeres. Así, por ejemplo, 67 \% de las mujeres descargan más de dos libros al año, mientras que este hecho sólo afecta a $60 \%$ de los hombres. Por otro lado, existe un mayor porcentaje de hombres $(31 \%)$ que de mujeres $(24 \%)$ que no descarga ningún libro. De nuevo el cruce de variables confirma los datos obtenidos en las Figuras 1 y 2. 
Mujeres

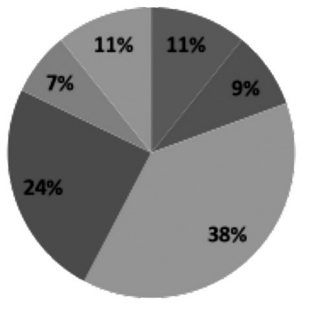

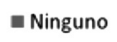

Uno

De dos a cuatro

De cinco a siete

De ocho a diez

- Más de diez
Hombres

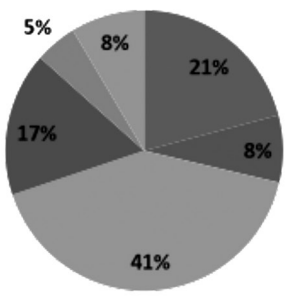

Figura 7. Compra de libros al año por sexo Fuente: elaboración propia a partir de la encuesta

El porcentaje de encuestados que se ve obligado a leer es bastante bajo en ambos sexos, aunque es $3 \%$ mayor en los hombres (Figura 8). En el caso de los encuestados que leen para aprender, el porcentaje es $13 \%$ mayor en hombres que en mujeres. Se observa, pues, un alto porcentaje de motivos utilitarios en la lectura. El cruce de variables confirma los datos expuestos a lo largo del presente apartado.

Aunque la mayoría de los estudiantes se limita a leer en español, hay $12 \%$ más de mujeres que lee en otro idioma. Conviene advertir que la presencia de un mayor número de alumnas en los grados relacionados con el aprendizaje de idiomas condiciona esta estadística.

El sexo no condiciona el uso de las redes sociales, ya que prácticamente casi todos los estudiantes las usan.

Es preocupante que alrededor de $17 \%$ de los alumnos universitarios extremeños no lea nada en su tiempo libre. Entre quienes leen, que son la mayoría, el porcentaje es sensiblemente mayor en las mujeres.
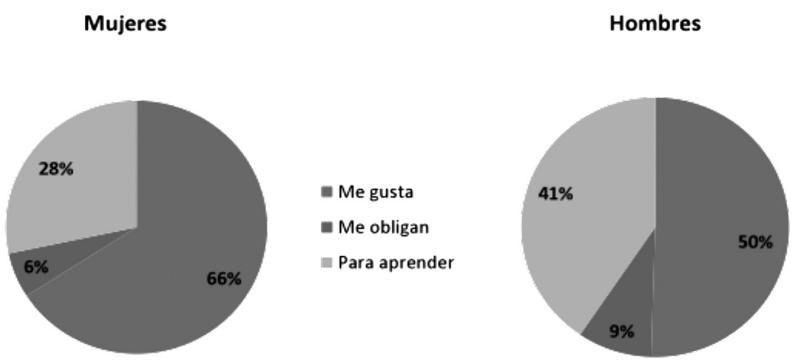

Figura 8. Motivo de la lectura por sexo Fuente: elaboración propia a partir de la encuesta 


\section{Otras variables (facultades, grados, máster)}

\section{Campo de estudio}

Para la realización de estas gráficas hemos agrupado a los alumnos por facultades dentro de grandes áreas de conocimiento, lo cual condiciona la estructura de las gráficas. Esta agrupación ficticia por áreas de conocimiento, que obedece únicamente a cuestiones de localización física de los estudiantes, tan sólo sería correcta si se alude a la planificación académica, en el caso de asociar a los alumnos de grado exclusivamente. La congregación de facultades por ramas se debe a la búsqueda de una significación estadística necesaria para la representación gráfica y cartográfica de los datos, así como para llegar a conclusiones realmente relevantes estadísticamente al contar con al menos 25 respuestas por ítems reflejados en las diferentes cuestiones. Así pues, la agrupación de facultades que se propone es la siguiente:

- Ingenierías y Ciencias: Escuela de Ingeniería Industriales, Escuela de Ingenierías Agrarias, Escuela Politécnica y Facultad de Ciencias.

- Ciencias Sociales y Jurídicas: Facultad de Ciencias de la Documentación y la Comunicación, Facultad de Ciencias del Deporte, Facultad de Ciencias Económicas y Empresariales, Facultad de Derecho, Facultad de Educación, Facultad de Estudios Empresariales y Turismo, y Facultad de Formación del Profesorado.

- Ciencias de la Salud: Facultad de Enfermería y Terapia Ocupacional, Facultad de Medicina y Facultad de Veterinaria.

- Humanidades: Facultad de Filosofía y Letras.

Las facultades de Ingenierías y Ciencias obtienen los resultados más bajos, con más de $30 \%$ de sus estudiantes encuestados que no lee ningún libro. El área de Humanidades resulta, en cambio, la que mejores resultados presenta, tanto en el rango de quienes leen entre dos y cuatro libros como en el rango de quienes leen más de cuatro. Sólo $10 \%$ de alumnos de esta facultad afirma no leer ningún libro al mes (Figura 9). 


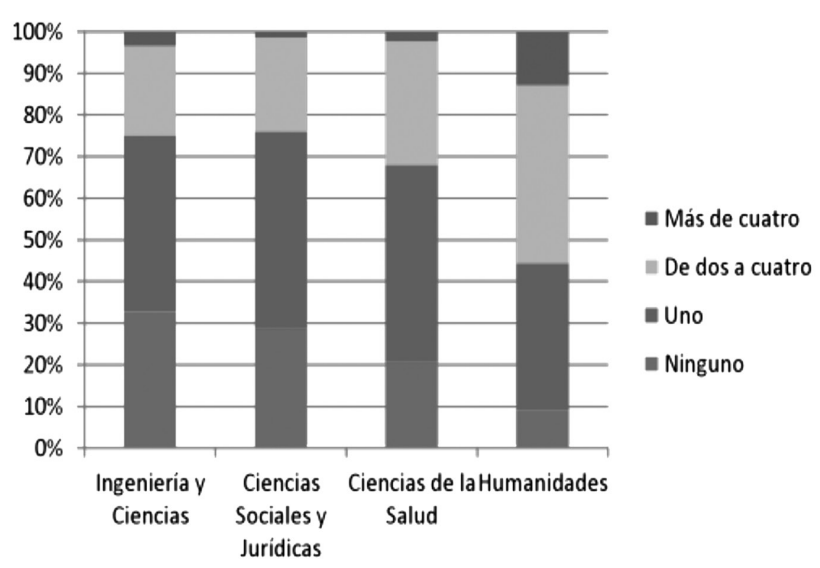

Figura 9. Libros leídos al mes según campo de estudio Fuente: elaboración propia a partir de la encuesta

$\mathrm{Al}$ igual que en el caso anterior, los porcentajes más bajos los presentan los estudiantes de Ingenierías y de la Facultad de Ciencias, y los más altos los de Humanidades, que obtienen siempre los mejores resultados en los rangos de más lectura (Figura 10). El cruce de variables, en este sentido, confirma que la mayor presencia de alumnado femenino en los grados de Humanidades y Ciencias Sociales puede estar relacionado con un mayor hábito de lectura.

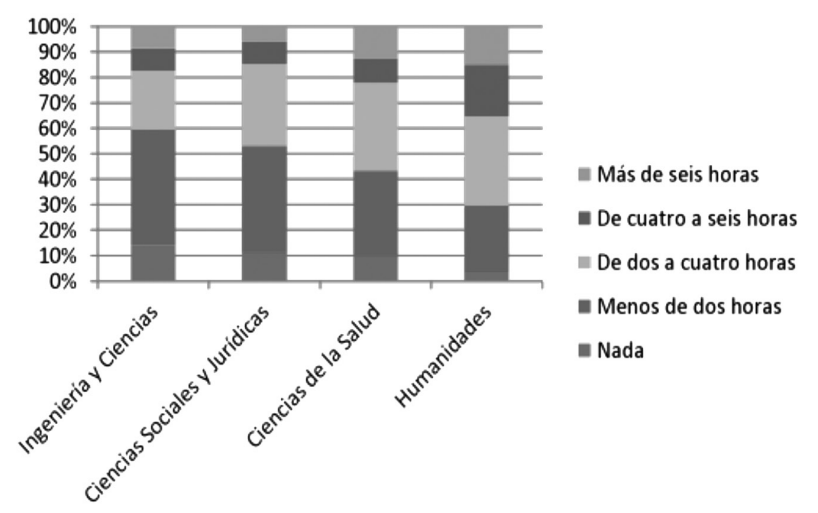

Figura 10. Tiempo de lectura a la semana según campo de estudio Fuente: elaboración propia a partir de la encuesta

Lo más llamativo de la Figura 11 resulta el alto porcentaje general de alumnos que afirma no pisar nunca la biblioteca. Los alumnos de Ciencias Sociales y 
Jurídicas constituyen el mayor grupo entre quienes no acuden jamás a la biblioteca; el mejor grupo en esta categoría vuelve a ser el de Humanidades. En cambio, se dan resultados muy dispares si atendemos a los grupos que acuden con más frecuencia. Así, entre quienes acuden más de cuatro veces se sitúan los de Ingeniería y Ciencias y los de Ciencias de la Salud.

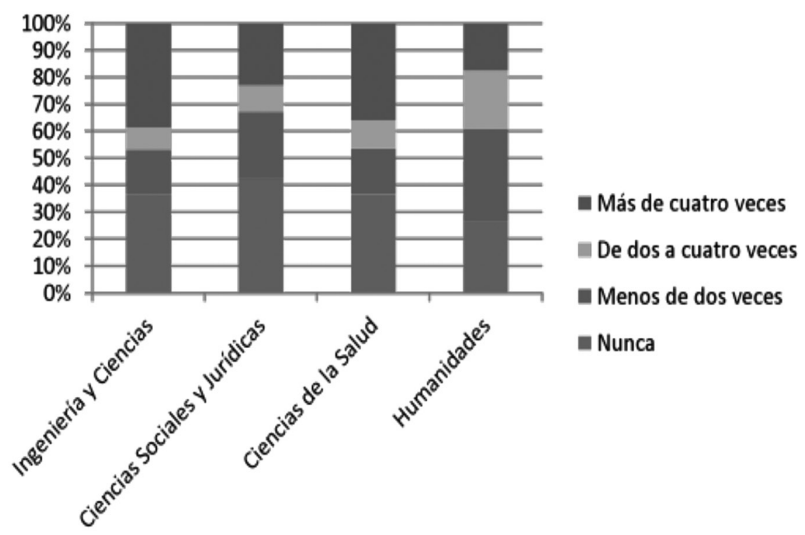

Figura 11. Frecuencia con que se acude a la biblioteca al mes según campo de estudio Fuente: elaboración propia a partir de la encuesta

Existe un porcentaje significativo de alumnos que afirma no comprar ningún libro al año, entre 7 y $18 \%$ según las áreas disciplinares. El rango de 2-4 libros comprados es el más concurrido (Figura 12). Los alumnos que menos libros compran son los de Ingenierías y Ciencias, mientras que los que más compran son los de Humanidades. Estos últimos constituyen, simultáneamente, el menor porcentaje entre quienes no compran ninguno, y el mayor porcentaje entre quienes compran más de 10.

Algo similar a lo que ocurre con la compra sucede con la descarga. Los que más libros descargan son los alumnos de Humanidades, con bastante diferencia, más de veinte puntos porcentuales sobre el segundo grupo, que serían los de Ingeniería y Ciencias, y muy por delante de los de Ciencias de la Salud, que es el grupo con menos hábito de descarga de libros. Llama la atención, por otro lado, que casi 30 \% de alumnos, tanto de Ingeniería y Ciencias como de Ciencias Sociales y Jurídicas, no descargue ni un solo libro. De nuevo, pues, el cruce de variables confirma la hipótesis de que la mayor presencia de alumnado femenino en los grados de Humanidades y Ciencias Sociales puede estar relacionado con mejores resultados en los hábitos lectores. 
Para finalizar, conviene indicar que la gran mayoría de universitarios lee durante su tiempo de ocio. El porcentaje nunca baja de $80 \%$. Con todo, se observa que los de Ciencias de la Salud y los de Humanidades tienen este hábito más adquirido, pues en ambos casos superan a los de Ingeniería y Ciencias y a los de Ciencias Sociales y Jurídicas en 10 puntos porcentuales.

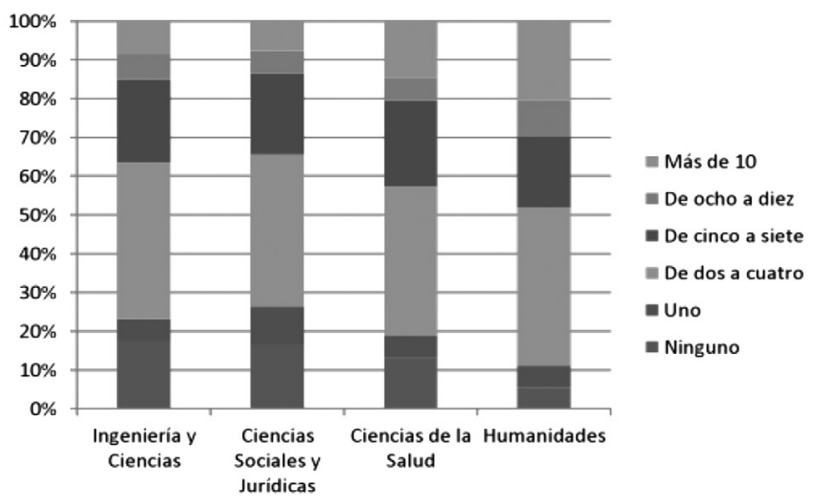

Figura 12. Compra de libros al año según campo de estudio Fuente: elaboración propia a partir de la encuesta

\section{Grado vs. posgrado}

El porcentaje de alumnos que no lee ningún libro al mes es menor en los cursos de posgrado, quizá por tener una mayor exigencia bibliográfica a la hora de la realización de trabajos y proyectos académicos (Figura 13).
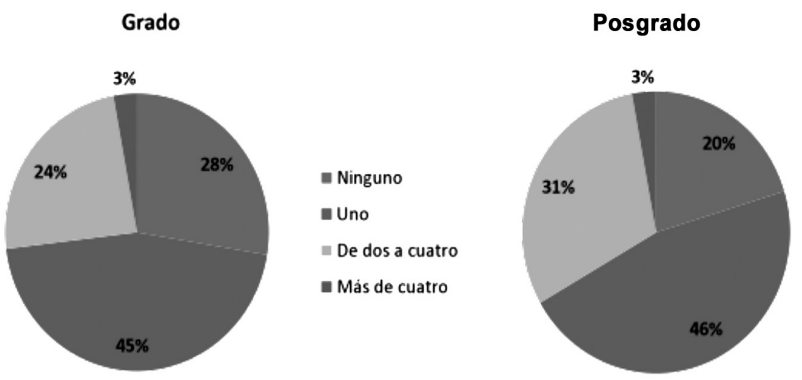

Figura 13. Libros leídos al mes según el nivel Fuente: elaboración propia a partir de la encuesta

Aunque en menor escala, estos resultados se corresponden con los anteriores, reflejando que los alumnos de posgrado dedican algo más de tiempo a 
la lectura que los de grado (Figura 14). También se observa claramente una mayor frecuencia de visitas a las bibliotecas entre los alumnos de posgrado.
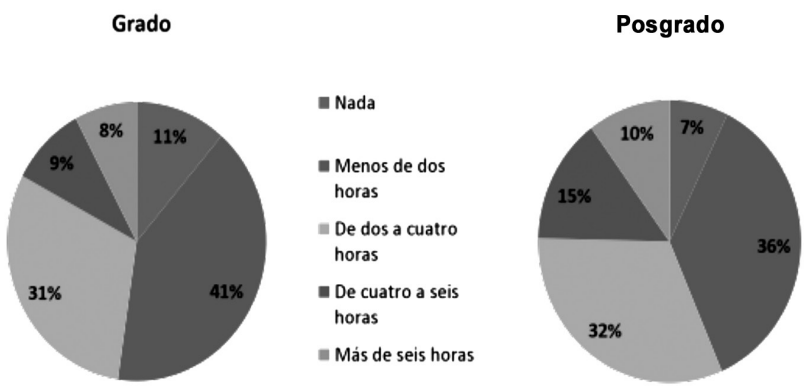

Figura 14. Tiempo de lectura a la semana según el nivel Fuente: elaboración propia a partir de la encuesta

En la Figura 15 los resultados son más parejos, incluso favorecen en algún punto a los alumnos de grado, como en el segmento de quienes no compran ningún libro al año, que supone $20 \%$ en el grado y $24 \%$ en posgrado. Al no concordar esta encuesta comparativa con las anteriores realizadas, no hay que descartar la idea de que ese menor índice en los alumnos de posgrado esté relacionado con un mayor esfuerzo económico por su parte ya que desaparecen prácticamente los estudios becados. Por otro lado, la comparación en la descarga de libros muestra unos resultados bastante similares, pues los distintos segmentos analizados se compensan, aunque favorecen mínimamente a los estudiantes de grado.

Grado
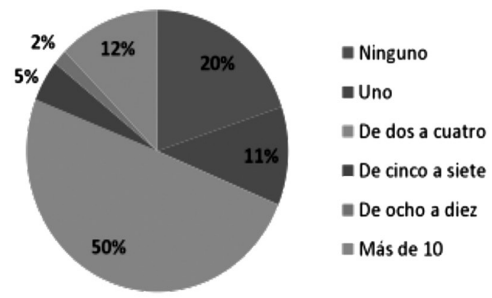

Figura 15. Número de libros comprados al año según el nivel

Fuente: elaboración propia a partir de la encuesta

Posgrado

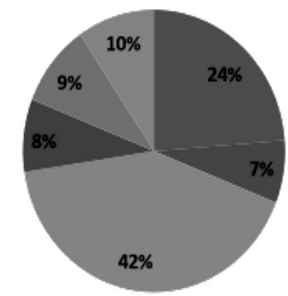

\section{.}

Aunque la mayoría de los alumnos lee sólo en español en ambos niveles, hay $10 \%$ más de alumnos en posgrado que lo hace también en otros idiomas. 
Al contrario de lo que sucede en otros niveles educativos, la lectura por obligación apenas existe en los niveles universitarios (7 \%), y además la poca que existe se va reduciendo desde el grado al posgrado (4\%). En otro orden de cosas, se aprecia un porcentaje mayor de alumnos de posgrado que lo hace con el deseo de aprender (39\%). Y, por otro lado, un porcentaje mayor, aunque no muy significativo, de alumnos de grado afirma leer por placer $(60 \%)$.

A la gran mayoría de universitarios les gusta leer en su tiempo libre ( $82 \%$ ), aunque es un hábito algo más extendido en estudiantes de posgrado (90 \%).

\section{Conclusiones}

Como primera conclusión y retomando las hipótesis de partida basadas en los estudios previos de Gilardoni (2006), puede afirmarse que los hábitos lectores de los estudiantes extremeños son muy similares a los de los estudiantes chilenos. Las líneas gruesas de las encuestas así lo confirman: pese a que una amplia mayoría aprecia la actividad de la lectura y reconoce sus bondades, casi un tercio de los alumnos no lee ningún libro al mes ni acude a las bibliotecas; la función principal de la lectura en estos niveles es fundamentalmente utilitaria, casi nunca está entre las primeras opciones como medio de ocio.

Como datos más destacados de nuestra encuesta, entre un cuarto y un tercio de los alumnos encuestados no lee ningún libro; sólo una cuarta parte los compra; la mitad de los estudiantes emplea menos de dos horas a la semana a la lectura; un cuarto de los encuestados lee también en otro idioma; dos tercios acude a las bibliotecas, más a estudiar que a sacar o consultar libros; se sigue prefiriendo la lectura en papel, si bien aumenta considerablemente la lectura en soportes digitales; leen más las mujeres que los hombres, los alumnos de posgrado que los de grado, los de Humanidades que los de Ciencias, Ingenierías y Tecnología; prácticamente la totalidad del alumnado usa redes sociales; los hombres tienden más a usar soportes digitales de lectura que las mujeres; los libros disciplinares son los más consultados; dentro de la ficción, los géneros literarios de misterio y aventuras son, en general, los más demandados; por último, el género lírico no atraviesa un buen momento en cuanto a las preferencias de lectura.

El cruce de variables confirma la hipótesis de que la mayor presencia de alumnado femenino en los grados de Humanidades y Ciencias Sociales está relacionada con unos mejores resultados generales en los hábitos de lectura. Así lo apuntan distintas variables como la lectura, las visitas a las bibliotecas 
universitarias, la compra y la descarga de libros. También ha interesado, desde el primer momento, comparar los resultados con otros estudios similares como el señalado de Gilardoni (2006).

Aunque el presente estudio parta originalmente de una iniciativa académica y constituya simplemente una aproximación estadística acerca de los comportamientos lectores de los estudiantes universitarios de una determinada región española (con las peculiaridades socioeconómicas señaladas), sus datos, el análisis de éstos y las conclusiones pueden resultar útiles de cara a implementar estrategias y políticas educativas y financieras (compra de determinado material bibliográfico, cambio de soportes, planes de fomento de lectura universitaria, rediseño de espacios bibliotecarios) por parte de la administración regional y de los órganos directivos de la Universidad.

\section{REFERENCIAS}

Aranda, José. 1975. "La encuesta de hábitos de lectura. Un ensayo de estudio de comportamiento". Estadística Española 68-69:53-78.

Arrieta, Beatriz, Judith T. Batista, Rafael D. Meza y Daniel Y. Meza. 2006. "La comprensión lectora y la redacción académica como centro del curriculum". Acción Pedagógica 15 (1): 94-98.

Bleotu, Vasile y Maria Doina. 2014. "Strategic Priorities for Education Funding". Procedia. Social and Behavioral Sciences. 116: 2231-2234.

Colomer, Teresa. 1993. "La enseñanza de la lectura. Estado de la cuestión". Cuadernos de Pedagogía 216: 15-18.

Comunidad Autónoma de Madrid. 2010. Hábitos de lectura y compra de libros en la Comunidad de Madrid. Fecha de consulta: 10 de noviembre de 2017. http://www. madrid.org/cs/Satellite?blobcol=urldata\&blobheader=application/pdf\&blobheadername1 $=$ Content-Disposition\&blobheadervalue1 $=$ filename $\% 3 \mathrm{DH} \%$ C3\%A1bitos+de+lectura $+2010+$ CM.pdf\&blobkey=id\&blobtable=MungoBl obs\&blobwhere $=1271881547480 \&$ ssbinary $=$ true

Corpas, María Dolores. 2014. "Análisis y evaluación de la comprensión lectora como lengua extranjera en Educación Secundaria Obligatoria”. Pbilologica Urcitana 11: 1-16.

Departamento de Cultura de la Generalitat de Cataluña. 2016. Hábitos de lectura y compra de libros en Cataluña. Fecha de consulta: 10 de mayo de 2018. https:// www.mecd.gob.es/dam/jcr:084ab0e5-ca0b-4d3a-a66e-e171506c7806/habits-lectura-catalunya-2016.pdf

Dobson, Alan, María Dolores Pérez y Richard Johnstone. 2010. Programa de educación bilingüe en España. Informe de evaluación. Fecha de consulta: 12 de enero de 2018. https://www.britishcouncil.es/sites/default/files/british-council-programa-educacion-bilingue-esp-informe-evaluacion.pdf

Echevarría, María Ángeles e Isabel Gastón. 2002. "Dificultades de comprensión lectora en estudiantes universitarios. Implicaciones en el diseño de programas de intervención”. Revista de Psicodidáctica 10:59-74. 
Fundación Germán Sánchez Ruipérez. 2008. Hábitos de lectura y compra de libros en Extremadura (14 a 24 años). Mérida: Plan de Fomento de la Lectura en Extremadura. Fecha de consulta: 10 de octubre de 2017. https://www.mecd.gob.es/dam/ jcr:12a6f0b0-14f5-4f85-bd9e-7944917dc92d/extremadura-2007.pdf

Fundación Germán Sánchez Ruipérez. 2010. Hábitos de lectura y compra de libros en Castilla-La Mancha, 2009. Junta de Castilla-La Mancha. Fecha de consulta: 10 de octubre de 2017. http://ccta.jccm.es/dglab2/public_dglab/aplicaciones/archivos/noticias/PresentacionEncuestaHabitosLectura2009.pdf

Furia, Donatella, Alina Castagna, Nicola Mattoscio y Domenico Scamuffa. 2010. "Education and labour market in the age of globalisation: some evidence for EU27”. Procedia. Social and Behavioral Sciences 9: 1140-1144.

Gil, Javier. 2009. "Hábitos y actitudes de las familias hacia la lectura y competencias básicas del alumnado". Revista de Educación 350: 301-322.

Gilardoni, Claudia. 2006. "Universitarios y lectura”. Calidad de la educación 25: 215-239.

Goksu, Alper y Donka Gungor. 2015. "A Comparative Analysis of Higher Education Financing in Different Countries". Procedia. Economics and Finance 26: 1152-1158.

Grecu, Mihaela, Emilia Titan y Elena Druica. 2015. "Investigating the Costs of Education in the European Union”. Procedia. Economics and Finance 23: 808-817.

Gremio de Libreros de Madrid. 2016. Mapa de librerías. Observatorio de la librería en España: Año 2015. Madrid: Observatorio de la Librería de CEGAL-MECD.

Grigorescu, Carmen. J. 2012. "Decision of Investment in Education- Necessity or Constraint?" Procedia. Social and Behavioral Sciences 46: 5520-5522.

Izquierdo, Belén. 2016. "Estrategias metacognitivas y recursos tecnológicos utilizados por estudiantes universitarios de español como segunda lengua”. Ocnos 15 (1): 149-164. http://dx.doi.org/10.18239/ocnos_2016.15.1.958

Junta de Extremadura. 2017. Atlas socioeconómico de Extremadura. Fecha de consulta: 11 de octubre de 2017. http://estadistica.gobex.es/web/guest/atlas-socieconomico-de-extremadura

López, Jesús y José A. Faíña. 2014. "Rhomolo and other methodologies to assess The European Cohesion Policy”. Investigaciones regionales 29: 5-13.

Martín, María. E. 2011. "Leer y escribir en la universidad: tarea pendiente". Álabe 4: 1-21. http://dx.doi.org/10.15645/Alabe.2011.4.5

Martos, Eloy. 2010. "De la República de las Letras a Internet: de la Ciudad Letrada a la cibercultura y las tecnologías del S. XXI". Álabe 1: 2-16. http://dx.doi. org/10.15645/Alabe.2010.1.3

Mate, María L. y Richard Harris. 2014. "Differential empirical innovation factors for Spain and the UK". Research Policy 43(2): 451-463. https://doi.org/10.1016/j.respol.2013.10.013

Millán, José A. (coord.). 2017. La lectura en España. Informe 2017. Madrid: Federación de Gremios de Editores de España.

Ministerio de Educación, Cultura y Deporte. 2001. Evaluación de la enseñanza y aprendizaje de la lengua inglesa. Educación secundaria obligatoria 2001. Informe fnal. Fecha de consulta: 21 de diciembre de 2017. https://www.mecd.gob.es/ $\mathrm{dctm} /$ ievaluacion/nacional/13evaluacion-de-la-ensenanza-y-el-aprendizaje-de-la-lengua-inglesa.pdf?documentId=0901e72b80110ded 
Ministerio de Educación, Cultura y Deporte. 2013. Nube de lágrimas. Club de lecturas en la nube; E-book +18 -40. Los lectores y los libros electrónicos; y Hábitos de lectura y compra de libros en España (2012). Fecha de consulta: 10 de octubre de 2017. http://www.mecd.gob.es/cultura-mecd/areas-cultura/libro/mc/observatoriolect/redirige/estudios-e-informes/elaborados-por-otras-entidades-con-la-colaboracion-del-ministerio/lectura.html

Ministerio de Educación, Cultura y Deporte. 2014. La participación de las familias en la educación escolar. Fecha de consulta: 2 de noviembre de 2017. http://www. mecd.gob.es/dctm/cee/publicaciones/estudioparticipacion/estudioparticipacion.pdf? documentId=0901e72b81b45e35

Ministerio de Educación, Cultura y Deporte. 2015. Encuesta de bábitos y prácticas culturales en España. 2014- 2015. Madrid: Ministerio de Educación, Cultura y Deporte.

Molina, Leandro. 2006. "Lectura y educación: los hábitos de lectura y su repercusión académica en Educación Secundaria Obligatoria”. Ocnos 2: 105-122. http://dx. doi.org/10.18239/ocnos_2006.02.07

Moreno, Celia, Fernando Guzmán y Eduardo García. 2017. “Los hábitos de lectura y escritura en los estudiantes de Educación Primaria: un análisis Dentro y Fuera de la escuela". Porta Linguarum, Monograph 2: 117-137.

Moret, Zulema. 1999. Animar a escribir para animar a leer. Salamanca: Fundación Germán Sánchez Ruipérez.

Muñoz, José M. y Azucena Hernández. 2011. “Hábitos lectores de los alumnos de la ESO en la provincia de Salamanca. ¿Son el género y el entorno factores diferenciales?" Revista de Educación 354, 605-628. doi: http://dx.doi.org/ 10.4438/1988-592X-RE-2011-354-013

Muñoz, Rosa y Lidia Taillefer. 2014. "Análisis de habilidades de lectoescritura y de hábitos de estudio en inglés como L2”. Porta Linguarum 22: 219-235.

Nieto, Ester. 2018. "Adquisición de la lectura en L1 en programas bilingües de Educación Primaria. Un estudio comparativo”. Ocnos 17 (1): 43-54. http://dx.doi. org/10.18239/ocnos_2018.17.1.1471

Nieto, Ana y José L. Gurría. 2005. "Análisis de la población de los programas de desarrollo rural en Extremadura mediante sistemas de información geográfica”. BIBLID 36: 479-495.

Nieto, Ana y Gema Cárdenas. 2015. "El Método LEADER como política de desarrollo rural en Extremadura en los últimos 20 años (1991-2013)”. Boletín de las Asociación de Geógrafos Españoles 69: 139-162.

Observatorio de la lectura y el libro. 2015. Panorámica de la edición española de libros 2015. Análisis sectorial del libro. Madrid: Ministerio de Educación, Cultura y Deporte (MECD).

Observatorio de la lectura y el libro. 2016. El libro digital. Aproximación a la realidad de las editoriales presentes en LIBER 2015. Madrid: MECD.

Observatorio de la lectura y el libro. 2017. El sector del libro en España. Madrid: MECD.

Olaziregi, María J. 2000. "Aproximación sociológica a los hábitos de lectura de la juventud vasca”. BIBLID 18: 79-93.

OCDE (Organización para la Cooperación y el Desarrollo Económicos). 2016. Programme for International Students Assessment, PISA, 2015. Madrid: MECD. 
Poulain, Martine (ed.). 1988. Pour une sociologie de la lectura. Paris: Editions du Cercle de la Librairie.

Ramajo, Julián, Miguel Á. Márquez y Francisco J. Miguel. 2014. "Economic impact of the European Funds in Extremadura during the period 2007-2013”. Investigaciones regionales 29: 113-128.

Ramírez, Elsa M. (coord.). 2015. Tendencias de la lectura en la universidad. México: Universidad Nacional Autónoma de México.

Riestra, Dora. 1999. "Reenseñar la escritura a estudiantes universitarios". Infancia y Aprendizaje 88: 43-56.

Serna, Maite, Arantzazu Rodríguez y Vavier Etxaniz. 2017. "Biblioteca escolar y hábitos lectores en Educación Primaria”. Ocnos 16 (1): 18-49. http://dx.doi. org/10.18239/ocnos_2017.16.1.1205

Para citar este texto:

Pérez Parejo, Ramón, Álvaro Gutiérrez Cabezas, José Soto Vázquez, Francisco Javier Jaraíz Cabanillas y José Antonio Gutiérrez Gallego. 2019. "Hábitos de lectura de los estudiantes de la Universidad de Extremadura (España). Aproximación estadística”. Investigación Bibliotecológica: archivonomía, bibliotecología e información 33 (79): 119-147.

http://dx.doi.org/10.22201/iibi.24488321xe.2019.79.57980 